\title{
Acoustic analysis of compensatory articulation in children
}

Shari R. Baum, and William F. Katz

Citation: The Journal of the Acoustical Society of America 84, 1662 (1988); doi: 10.1121/1.397181

View online: https://doi.org/10.1121/1.397181

View Table of Contents: https://asa.scitation.org/toc/jas/84/5

Published by the Acoustical Society of America

\section{ARTICLES YOU MAY BE INTERESTED IN}

Effects of rate and bite block manipulations on kinematic characteristics of children's speech

The Journal of the Acoustical Society of America 81, 747 (1987); https://doi.org/10.1121/1.394843

Differences in fricative production between children and adults: Evidence from an acoustic analysis of / $/ /$ and /s/ The Journal of the Acoustical Society of America 83, 229 (1988); https://doi.org/10.1121/1.396425

Incomplete compensation to articulatory perturbation

The Journal of the Acoustical Society of America 97, 1865 (1995); https://doi.org/10.1121/1.412060

Compensation strategies for the perturbation of the rounded vowel [u] using a lip tube: A study of the control space in speech production

The Journal of the Acoustical Society of America 98, 2428 (1995); https://doi.org/10.1121/1.413277

Time-varying spectral change in the vowels of children and adults

The Journal of the Acoustical Society of America 108, 1856 (2000); https://doi.org/10.1121/1.1289363

Development of a test of speech intelligibility in noise using sentence materials with controlled word predictability

The Journal of the Acoustical Society of America 61, 1337 (1977); https://doi.org/10.1121/1.381436

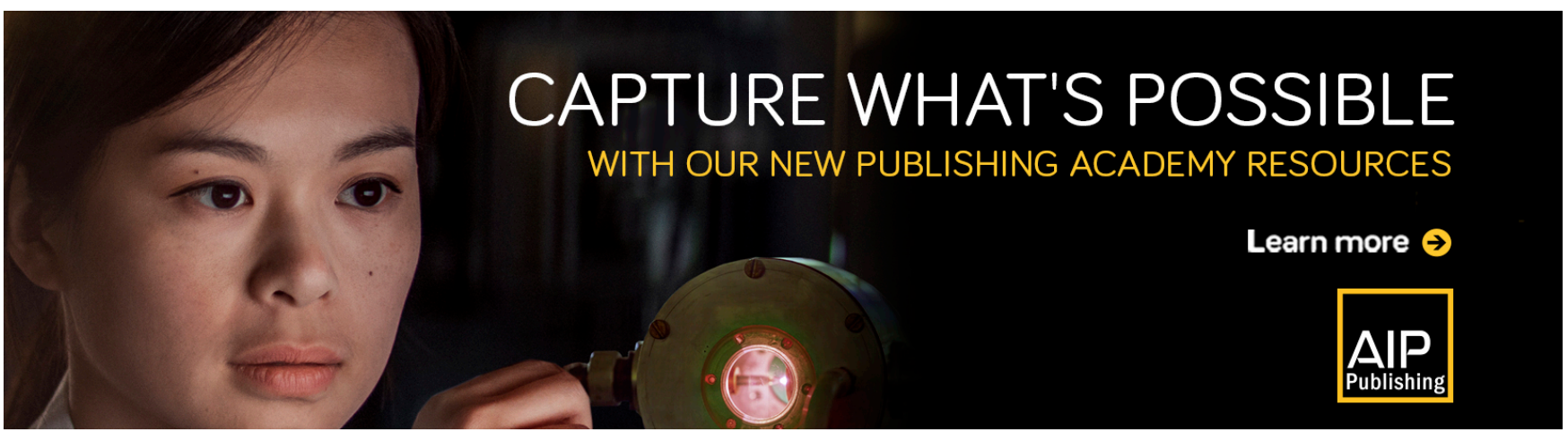




\title{
Acoustic analysis of compensatory articulation in children
}

\author{
Shari R. Baum ${ }^{\text {a) }}$ \\ Department of Cognitive and Linguistic Sciences, Box 1978, Brown University, Providence, Rhode Island \\ 02912 \\ William F. Katz \\ Department of Psychiatry, M-031P University of California at San Diego, La Jolla, California 92093
}

(Received 11 April 1988; accepted for publication 12 July 1988)

\begin{abstract}
A study was undertaken to explore the effects of fixing the mandible with a bite block on the formant frequencies of the vowels [ $\mathrm{i}$ a $\mathrm{u}$ ] produced by two groups of children aged 4 and 5 and 7 and 8 years. Vowels produced in both normal and bite-block conditions were submitted to LPC analysis with windows placed over the first glottal pulse and at the vowel midpoint. For both groups of children, no differences were found in the frequencies of either the first or second formant between the normal and bite-block conditions. Results are discussed in relation to theories of the acquisition of speech motor control.
\end{abstract}

PACS numbers: 43.70.Ep, 43.70.Aj

\section{INTRODUCTION}

In recent years, substantial research has focused upon the study of speech motor control. One useful technique has been to investigate speech production during conditions in which there is a perturbation (either expected or unexpected) of some part of the articulatory apparatus. Perturbation studies have allowed for the examination of a phenomenon known as "compensatory articulation," i.e., the achievement of a goal or target production involving compensatory movement by the nonperturbed articulators. The results of these studies have suggested that there is some type of invariant goal in the production of a given speech sound and that variable muscle activations serve to reach that goal (Abbs, 1986; Folkins and Linville, 1983; Folkins and Zimmermann, 1982; Gracco and Abbs, 1985; Hughes and Abbs, 1976; Kelso and Tuller, 1983a,b). These hypotheses have been referred to as "motor equivalence" (Hebb, 1949, MacNeilage, 1970 ) or "equifinality" (Kelso and Tuller, 1983b).

The phenomenon of compensatory articulation has been studied using articulatory (as noted above), acoustic, and perceptual analyses. These studies have answered a number of important questions about the muscle synergies involved in compensatory speech behavior, their acoustic correlates, and the corresponding perceptual implications for listeners. With regard to acoustic studies, most analyses have been conducted investigating speech produced with and without fixation of the mandible by a bite block. One of the earliest of these experiments was conducted by Lindblom and Sundberg (1971). In this study, various sized bite blocks were used to fix the jaw, and the formant frequencies of subjects' vowel productions in both the normal and bite-block conditions were measured. Spectrographic evidence showed that, even at the first glottal pulse, before auditory feedback could occur, the vowel formants in the bite-block condition approximated those of the normal condition. These results

\footnotetext{
a) Requests for reprints should be sent to Shari R. Baum, Ph.D., School of Human Communication Disorders, McGill University, Montreal, Quebec H3G 1A8, Canada.
}

were surprising in light of an articulatory model for the production of vowel acoustics, which predicted that the first three vowel formants should demonstrate increases in frequency with an increase in jaw opening (Lindblom and Sundberg, 1971). The authors concluded that these data provided evidence of compensatory articulation which occurred immediately. These early results have been replicated in many studies, involving both acoustic and articulatory kinematic data (e.g., Fowler and Turvey, 1980; Gay et al., 1981; Lindblom et al., 1979; Lubker, 1979).

One possible means of gaining more information about issues in speakers' compensatory articulation abilities is to examine the developmental time course of these abilities in children. These data would shed light upon issues concerning early versus late aspects of speech development in children. If very young children show compensatory articulation abilities, this would suggest a relatively minimal role of experience in compensatory articulation development. If, on the other hand, young children demonstrate much more inconsistent or reduced compensatory abilities, it would suggest that this feature of articulatory control is acquired relatively late in development and is much more dependent upon experience.

There has been a good deal of evidence which demonstrates that much articulatory control is acquired gradually. For instance, Eguchi and Hirsh (1969) found much more variability in vowel formant frequency production in children up to age 11, while Kewley-Port and Preston (1974) and Macken and Barton (1980) found increased variability in production of voice onset time in children through age 8. Other durational and acoustic-phonetic measures have also been shown not to approximate those of adult productions until neuromuscular and cognitive maturation proceed (e.g., Konefal et al., 1982; Kubaska and Keating, 1981; Sereno et al., 1987; Smith, 1977). In addition, articulatory measurements, particularly with regard to the lip-opening gesture (e.g., associated with production of [a]), have indicated a similar pattern of instability until approximately 
age 10 (e.g., Sharkey and Folkins, 1985; Watkin and Fromm, 1984).

Several experiments have focused specifically on the compensatory articulation abilities of children. Oller and MacNeilage (1983) conducted a bite-block experiment with two children, aged 4 and 8 . The children were required to repeat tape-recorded models of the vowels $[i, \mathfrak{x}]$ in both normal and fixed-jaw conditions. Using narrow-band spectrograms, the researchers found it difficult to isolate the formant frequencies of the vowels due to the high fundamental frequencies of the children's speech (cf. Peterson and Barney, 1952). Oller and MacNeilage thus calculated a resonance measure $(R 2)$ rather than directly measuring formant peaks. Inconsistent $R 2$ values measured at the vowel's midpoint were found even within conditions for the younger child. The 8-year-old, while producing stable $R 2$ measures within conditions, varied a great deal across the conditions, with the formants of the fixed-mandible vowels considerably lower than those of the free-mandible vowels. A perceptual test incorporating both free and fixed-jaw vowels yielded fairly accurate results though, prompting the authors to propose that the children were compensating by means other than formant frequency approximation.

More recently, Smith and McLean-Muse (1987a) conducted a study of the effects of a bite block on the articulatory patterns of children's speech. These researchers found net peak velocity and displacement patterns of the lip and jaw similar to those found with adult subjects in three groups of children aged 4 and 5, 7 and 8, and 10 and 11 years. Despite increased variability, the authors claim that "the ability to produce speech under experimental conditions such as these is apparently acquired by normally developing children by at least 4-5 years of age" (p. 752). It remains unclear what the acoustic consequences of these compensatory articulation patterns are. In particular, the spectral energy distribution, as determined by the vocal tract area function, serves as an important perceptual cue to phoneme identity. It is, therefore, crucial to explore the acoustic patterns associated with both perturbed and unperturbed productions. In addition, in contrast to the results presented by Oller and MacNeilage (1983) and Smith and McLean-Muse (1987a), Gibson and McPhearson (1980) found that children 6 to 7 years of age did not show complete compensation for biteblock manipulations when producing several vowels of Swedish. Like Oller and MacNeilage (1983), these researchers had difficulty isolating the formant frequencies of the children's speech using spectrographic analyses. Those formant values that could be derived were relatively close in both normal and bite-block conditions. However, in a perception study, Gibson and McPhearson found that some of the vowels produced in the bite-block condition were less accurately transcribed than those in the normal condition; the authors conclude that "the children appeared at least in a number of instances to be either not compensating for the presence of the bite block, or more likely to be only partially compensating for it" (Gibson and McPhearson, 1980; p. 42). They further suggest that the "'neurophysiological targets' must be language-dependent and learned aspects of speech motor control" (p. 42).
It thus appears that the question of compensatory articulation abilities in children is still far from settled. In the present study, we pursue these issues by determining whether a larger group of young subjects than that used in the Oller and MacNeilage (1983) study would demonstrate acoustic evidence of compensatory articulation. If young children exhibit compensatory abilities, as suggested by Smith and McLean-Muse (1987a), it might suggest that the functional organization of the speech musculature is specified quite early in development. If, on the other hand, compensatory articulation abilities are only gradually acquired, it might suggest a relatively late fine tuning of articulatory skills necessary to achieve given acoustic targets (Gibson and McPhearson, 1980). The present study represents an attempt to address this issue through acoustic analyses.

\section{METHOD}

\section{A. Subjects}

Five children in each of two age groups $(4 ; 0-5 ; 6$ years, $X=5 ; 2$ and 7;0-8;6 years, $X=8 ; 4)$ participated in this study. All subjects were selected from the UCSD Child Language Research Subject Pool. The children were monolingual speakers of English living in the San Diego area. In order to qualify for the study, subjects had to have shown no history of speech or language difficulties (as determined by a parent questionnaire). Children were also given a pretest screening by one of the researchers in order to insure that the subjects had no dental malocclusions or other oral cavity anomalies.

\section{B. Materials and procedure}

Subjects were tape recorded using a Nagra 4.2 recorder and high-quality microphone, producing the vowels [i], $[u]$, and [a] in response to a tape-recorded model (adult male speaker). The children were encouraged to produce the best approximation possible. Each vowel was produced a total of 14 times in each condition (normal and bite block). Normal and bite-block productions were randomly distributed for a total of 84 tokens per subject. Two repetitions were elicited after each recorded model production. These two repetitions were analyzed separately to ascertain whether any potential order effects emerged. ${ }^{1}$

Bite blocks were constructed out of dental material, as suggested by Netsell (1985). It was initially attempted to have subjects create molar impressions in the softened dental impression compound in order to prevent bite-block slippage. However, this technique proved unworkable for the younger children and had to be modified. Instead, small blocks (approximately $1.8 \times 0.8 \times 1.5 \mathrm{~cm}$ ) were fashioned out of the dental impression material. The exact size of each block was individually tailored for each subject in order to insure a vertical distance of $12 \mathrm{~mm}$ between the central incisors when held between the molar and premolar teeth. The porous surface of each bite block proved to be quite adequate for preventing slippage during production. The subjects were assisted in bite-block placement by one of the researchers. Care was taken to insure that the bite block did not protrude into the oral cavity and obstruct the tongue body. 


\section{Acoustic analyses}

Vowel tokens were digitized using a PDP 11/34 computer at a rate of 20k samples per second with 10-bit quantization and a 9-kHz low-pass filter. Linear predictive coding (LPC) analyses were performed using a 24-pole network and a variable-sized full Hamming window placed over the first glottal pulse of the vowel. A second LPC analysis was performed at the vowel's midpoint, using a $25.6-\mathrm{ms}$ full Hamming window. First and second formant frequencies were derived from the LPC analyses. ${ }^{2}$ Based on previous data (e.g., Eguchi and Hirsh, 1969; Pickett, 1980), the highest amplitude peak in the frequency range $300-650 \mathrm{~Hz}$ was considered to be $F 1$ for [i] and [u], and in the range 800-
$1400 \mathrm{~Hz}$ for [a]. Similarly, $F 2$ was defined as the highest amplitude peak found in the frequency range $2800-3700 \mathrm{~Hz}$ for [i] , and $1300-2000 \mathrm{~Hz}$ for [u] and [a]. If no peak occurred within these ranges, no formant value was calculated for that token. Precise formant accuracies cannot be provided, but are estimated to be within $25 \mathrm{~Hz}$ of the calculated values.

\section{RESULTS}

Mean $F 1$ and $F 2$ frequencies for each vowel for each subject were calculated separately for the two window placements (first glottal pulse and vowel midpoint) and for first and second repetitions in the normal and bite-block condi-

TABLE I. Mean $F 1$ and $F 2$ values (in $\mathrm{Hz}$ ) for each subject for each vowel in normal (N) and bite-block (BB) conditions.

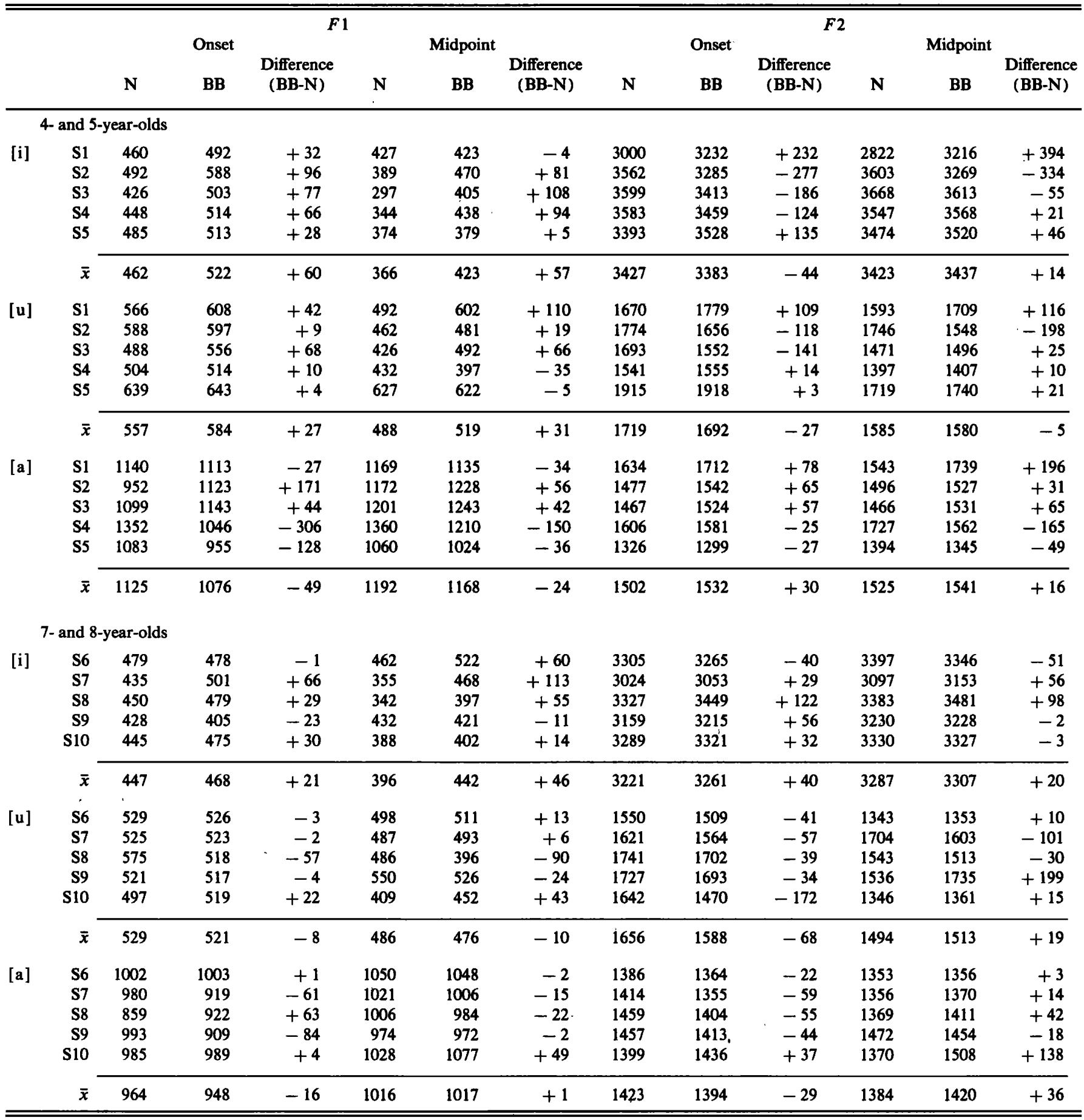


tions. In order to determine whether any repetition effect would emerge, two analyses of variance (ANOVA) with three within-subject factors (repetition $\times$ vowel $\times$ window) and one between subjects factor (age group) were conducted on the bite-block data only, for $F 1$ and $F 2$ values separately. Both ANOVAs indicated no significant main effects for repetition [ for $F 1, F(1,8)=5.09$ n.s.; for $F 2, F(1,8)=0.01$ n.s. ] nor any interactions with the repetition variable. The data from the first and second repetitions were thus pooled for further analysis.

Table I presents the averaged $F 1$ and $F 2$ data for each subject and the means for the two groups of subjects ( 4 and 5 years and 7 and 8 years) in both normal and bite-block conditions, as measured at the two window placements for each vowel. As is clear from the table, there were some differences between formant values measured in the normal and biteblock conditions. As an example, for the 4- and 5-year-old group, mean $F 1$ values (as measured at the vowel midpoint) for [i] were 366 and $423 \mathrm{~Hz}$ in the normal and bite-block conditions, respectively (a difference of $57 \mathrm{~Hz}$ ). Mean $F 2$ values for [i] were $3423 \mathrm{~Hz}$ in the normal condition and $3437 \mathrm{~Hz}$ in the bite-block condition-a difference of $14 \mathrm{~Hz}$. Similarly, for the 7-and 8-year-old subjects, $F 1$ (midpoint) frequencies for [i] were 396 and $442 \mathrm{~Hz}$ in the normal and bite-block conditions, respectively, yielding a difference of $46 \mathrm{~Hz}$. The $F 2$ values calculated for [i] were $3287 \mathrm{~Hz}$ in the normal condition and $3307 \mathrm{~Hz}$ in the bite-block condition (a
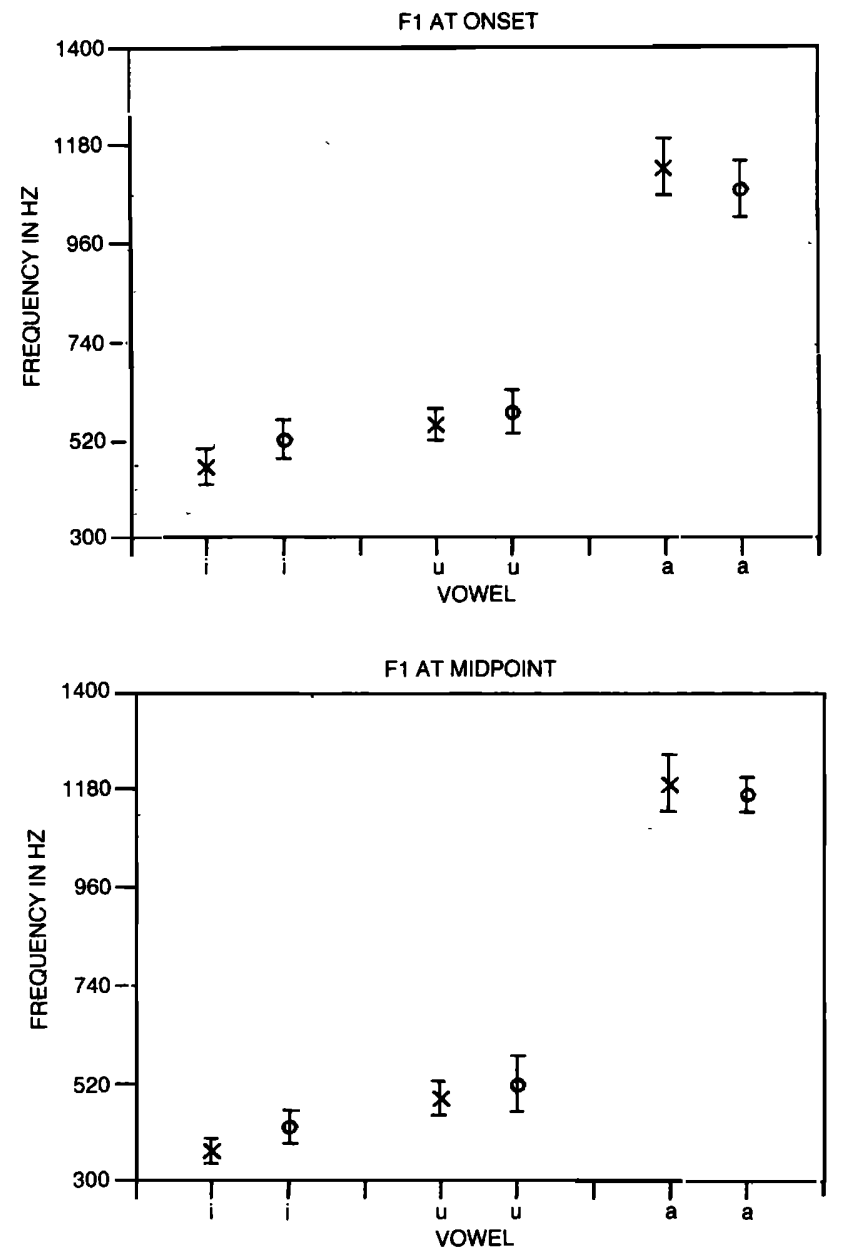

difference of $20 \mathrm{~Hz}$ ). In general, most mean frequency differences were small, ranging from $1 \mathrm{~Hz}(F 1$ of [a] at midpoint for 7-and 8-year-olds) to a maximum of $68 \mathrm{~Hz}(F 2$ of [u] at first glottal pulse for 7- and 8-year-olds). In fact, most of these differences fall within the difference limens $(d l s)$ for the given frequency ranges (Flanagan, 1955) and would thus be imperceptible. It should be noted, however, that, for certain subjects, atypically large frequency differences did emerge (e.g., $F 2$ for [i] for subjects $\mathbf{S} 1$ and $\mathrm{S} 2$ ). Moreover, the mean differences were, on average, no greater for the younger subjects than for the 7-and 8-year-olds. As expected, formant frequency values were, overall, somewhat higher for the younger subjects, often by as much as $100 \mathrm{~Hz}$ (particularly for $F 2$ values).

Figures 1 and 2 provide graphic representations of the mean data along with standard deviation values for the two subject groups, respectively. As may be noted, there was a slight tendency for the formants in the bite-block condition to be higher than in the normal condition, but the standard deviation values were comparable in both conditions. In addition, there was a tendency for standard deviation values to be higher for the younger group, as may be seen in a comparison of Figs. 1 and 2. Subjects' mean formant frequency values (for $F 1$ and $F 2$ values separately) were submitted to ANOVAs, including subject age group ( 4 and 5 years, 7 and 8 years), window placement (first glottal pulse, midpoint), vowel $(\mathrm{i}, \mathrm{a}, \mathrm{u})$, and condition (normal, bite block) as factors.
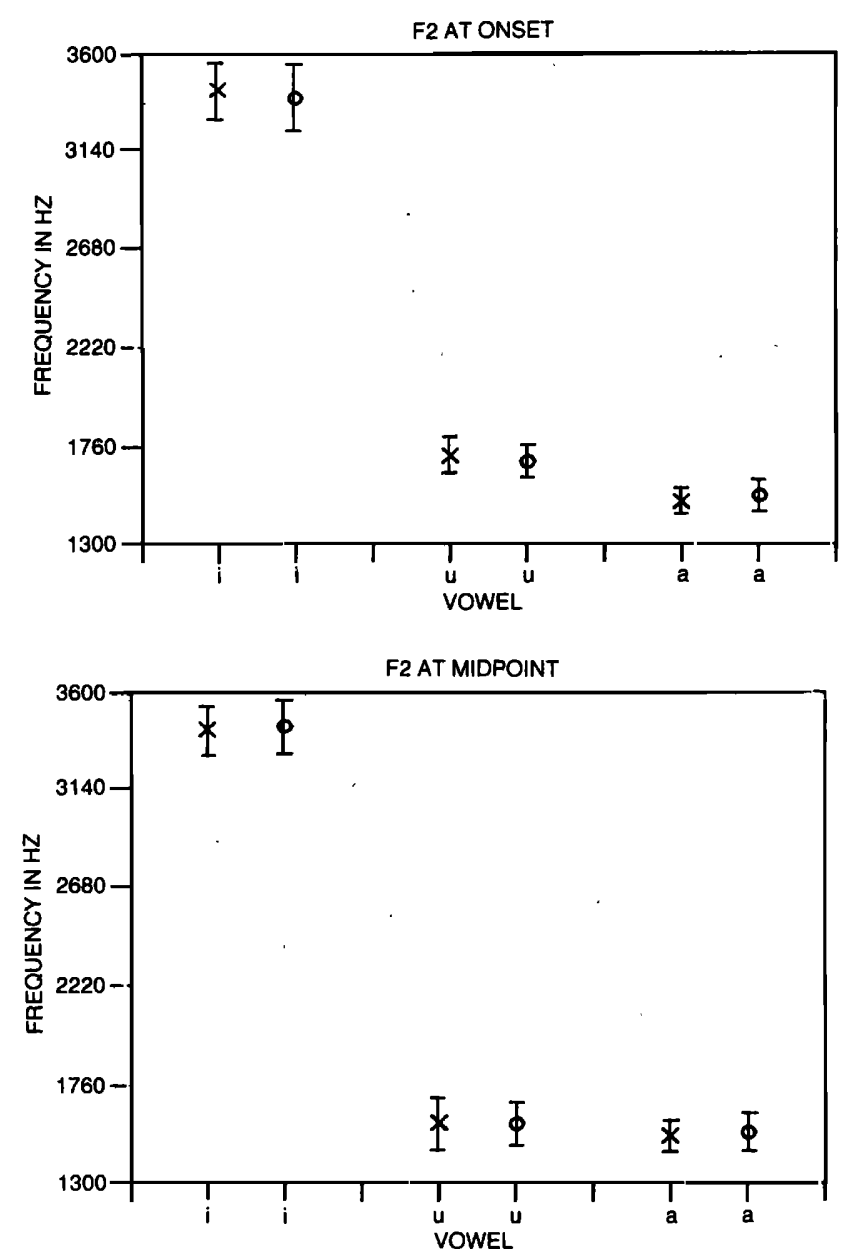

FIG. 1. Means and standard deviations of $F 1$ and $F 2$ frequencies in normal $(X)$ and bite-block $(O)$ conditions for vowels [i a u] produced by 4-to 5-year-old subjects. 
Both ANOVAs revealed significant main effects for subject group $[F 1: F(1,8)=36.20, p<0.001 ; F 2: F(1,8)=19.10$, $p<0.005$ ], confirming that, overall, $F 1$ and $F 2$ values were higher for the younger children (as expected). In addition, both analyses revealed significant main effects for vowel, as expected $[F 1: \quad F(2,16)=380.49, \quad p<0.001 ; \quad F 2$ : $F(2,16)=470.91, p<0.001]$. Importantly, neither ANOVA showed a significant main effect for condition (normal, bite block), nor any interactions of that variable with any other [ $F 1$, main effect: $F(1,8)=0.73$ n.s.; $F 2$, main effect: $F(1,8)=0.00 \mathrm{n} . \mathrm{s}$. ]. There was a tendency for the formant values measured at the first glottal pulse to differ from those measured at the midpoint of the vowel; however, these differences occurred in both the normal and bite-block conditions, suggesting that the differences were not due to the subject becoming accustomed to (or adjusting for) the bite block throughout the duration of the vowel.

Standard deviation values for $F 1$ and $F 2$ were also submitted to similar analyses of variance (group $\times$ condition $\times$ vowel $\times$ window). Results revealed a significant condition $\times$ vowel $\times$ window interaction for $F 1$ values $[F(2,16)=4.67, p<0.05]$. Planned Newman-Keuls post hoc statistics $(p<0.01)$ demonstrated no significant differ-
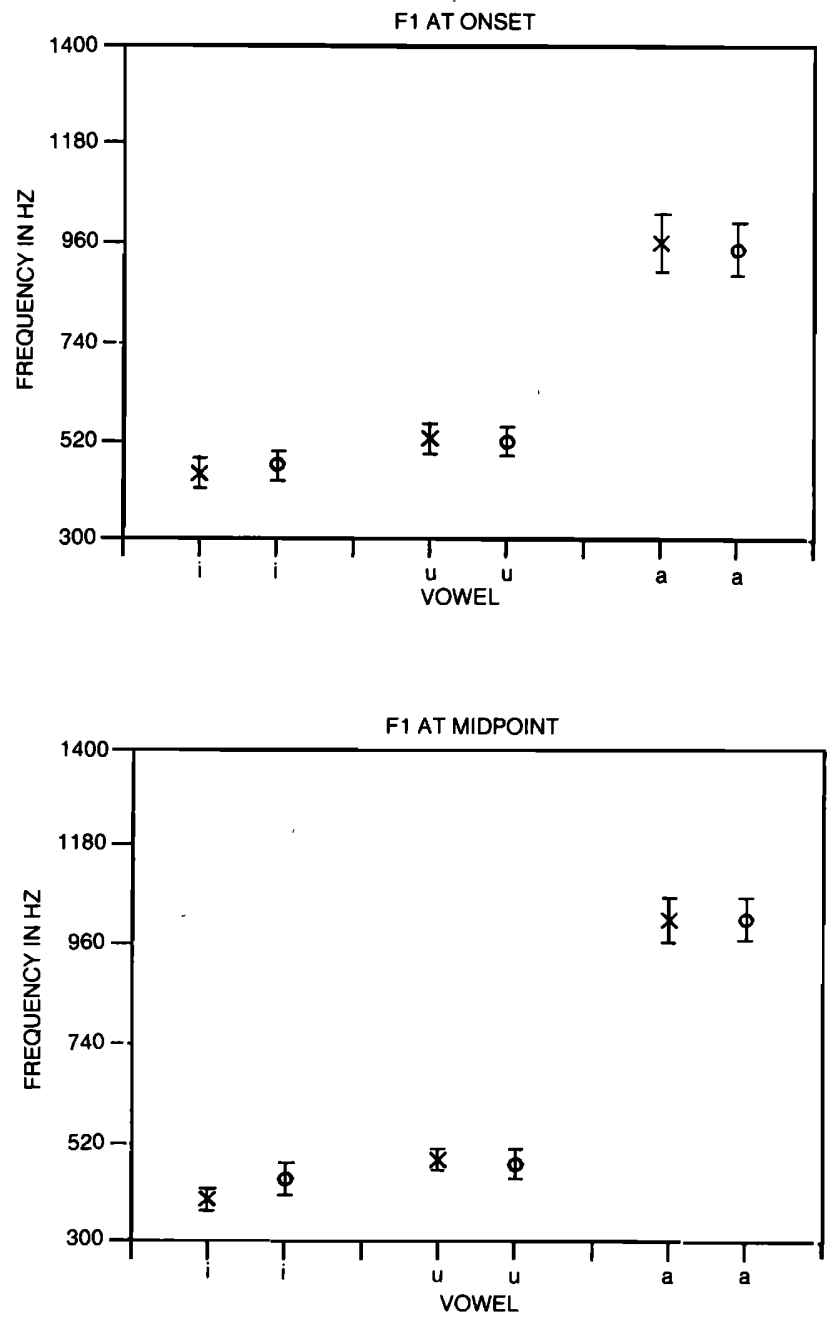

ences between $F 1$ standard deviation values in the normal and bite-block conditions. No significant main effects or interactions with condition were found for $\boldsymbol{F} \mathbf{2}$ standard deviation values. Interestingly, the exhibited trends for $F 1$ and $F 2$ standard deviation values to be higher for the younger subject group did not reach statistical significance.

\section{DISCUSSION}

The results of the present study suggest that by 4 to 5 years of age children are able to compensate for perturbation by a bite block by producing acoustically comparable vowel tokens, even as measured at the first glottal pulse of the vowel. These data are in accord with the articulatory data gathered by Smith and McLean-Muse (1987a) who found that 4and 5-year-old children demonstrated articulatory compensation for bite-block manipulations. Although the data are in contrast with those of Gibson and McPhearson (1980), several potential explanations may account for the discrepancy. First, as stated by Gibson and McPhearson (1980), the acoustic data that they present may not be very reliable and are subject to errors in measurement of up to $100 \mathrm{~Hz}$. An alternative hypothesis is that, given the more "crowded" vowel space in Swedish as compared to English, less variabil-
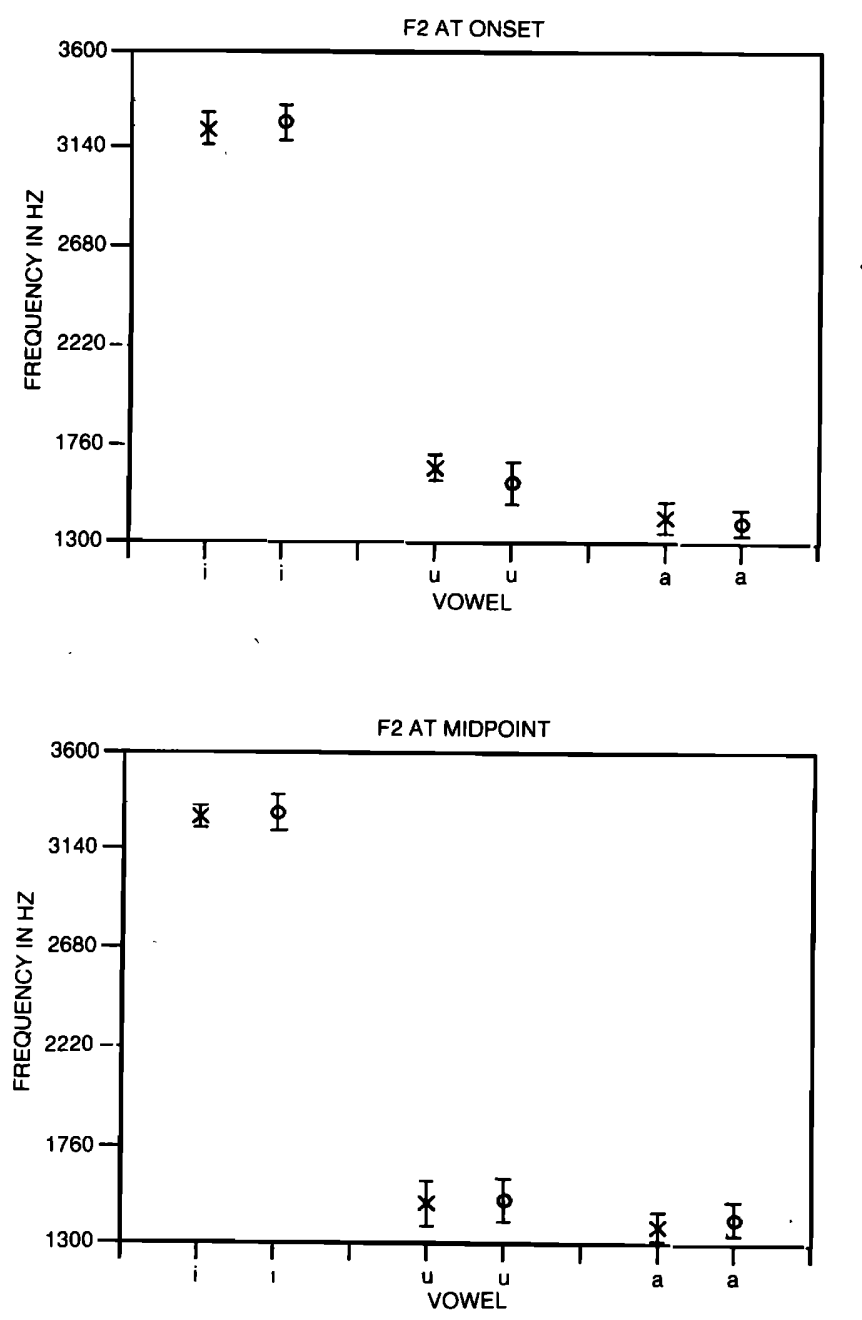

FIG. 2. Means and standard deviations of $F 1$ and $F 2$ frequencies in normal $(X)$ and bite-block $(O)$ conditions for vowels [i a u produced by 7 -to 8 -year-old subjects. 
ity in formant frequencies is permissible for accurate perceptual identification.

A second important finding in the current study was a tendency for the younger (4- and 5-year-old) subjects' productions to be somewhat more variable than the older subjects' (7-and 8-year-olds), consistent with previous research (e.g., Eguchi and Hirsh, 1969; Kewley-Port and Preston, 1974). Yet, the present results revealed no significant differences in formant frequency values in normal as compared to bite-block conditions for either group of children studied (4and 5-year-olds and 7- and 8-year-olds). Moreover, although differences were found between formant frequencies measured at the first glottal pulse of the vowel and those measured at the vowel's midpoint, the differences appeared in both normal and bite-block conditions. These results suggest that auditory feedback played no role in compensating for the presence of the bite block (i.e., that compensation occurred "immediately"). Formant frequencies calculated at the first glottal pulse of the vowel in the bite-block condition approximated those in the normal condition, as did those measured at the vowel's midpoint. In addition, vowels were repeated twice in succession; a comparison of $F 1$ and $F 2$ values in the first and second repetitions again revealed no significant differences, confirming that learning did not play an important role in compensation. It should be pointed out, however, that the articulatory perturbation was not unexpected and thus it cannot be determined whether the compensatory adjustments occurred "on-line" or at some advance planning stage.

An examination of standard deviation values in the two conditions also demonstrated no significant differences, suggesting that the children's vowel productions were not more variable in the bite-block than in the normal condition. It is important to note, though, that these conclusions are based on a failure to find significant differences between conditions and thus should be considered cautiously; that is, it is possible that the measure is simply not sensitive enough to discern differences. Alternatively, potential differences between vowel formants produced in the normal and bite-block conditions may be obscured due to the overall variability of the children's productions. Nevertheless, based on the present results and the results of previous studies (e.g., Lindblom et al., 1979; Smith and McLean-Muse, 1987a), it is our belief that the production of comparable $F 1$ and $F 2$ peaks in the normal and bite-block conditions does reflect compensatory articulation strategies present in these children.

The rapidity of compensation in the production of vowel targets has suggested to some that invariant phonetic goals are coded in terms of acoustically significant vocal tract area function features (Gay et al., 1981; Lindblom et al., 1979). Some investigators have suggested that "immediate" compensation might be accomplished through feed-forward mechanisms that allow the speaker to adjust for variations in initial conditions, rather than relying on auditory or proprioceptive feedback (Borden, 1979; Lindblcm et al., 1979). For instance, Kelso and Tuller (1983a) have shown that compensatory articulation occurs even when sources of auditory, proprioceptive, and tactile feedback are reduced or eliminated. In contrast, others have shown evidence that feedback may, in fact, be involved in perturbed productions, particularly when speakers are given opportunities to make repeated production attempts (Flege et al., 1988). The results of the current study do not demonstrate any improvement with repetition, but the role of feedback cannot be dismissed since both tactile and proprioceptive feedback were available to the subjects in this task. Auditory feedback, however, does not seem to be involved. That is, although auditory feedback has been shown to play a critical role in establishing accurate speech sound contrasts in children (e.g., Borden, 1979), it is less clear what role it plays in online speech motor control in older children and adults.

In several recent studies, it has been suggested that, although some compensatory articulation is evident in the speech of both adults and children, compensation is incomplete (Flege et al., 1988; Gibson and McPhearson, 1980; Smith and McLean-Muse, 1987b); that is, certain articulatory parameters are critical in order to produce perceptually acceptable speech sounds, while others may be less important in compensation. ${ }^{3}$ These claims are not unlike those of Lindblom et al. (1979), who contended that normal adult subjects compensate for fixation of the mandible by best approximating the normal vocal tract configuration at the acoustically salient points of constriction. These findings may be interpreted to suggest that subjects aim for an acoustic/perceptual goal and adjust their articulators accordingly (cf. Perkell, 1980).

The current results suggest that children as young as 4 or 5 years of age demonstrate such compensatory abilities, at least as far as certain perceptually critical acoustic cues are concerned. That is, at a time when normal vowel formants are still quite variable (Eguchi and Hirsh, 1969), the ability to compensate for fixation of the mandible is present. Although at first somewhat surprising, this finding is not altogether unexpected. First, although much articulatory control remains undeveloped at this age, a good deal of precision has been achieved. In addition, it is not uncommon for a child of even a younger age to speak with an object in his mouth (e.g., a piece of food). Thus, by age 4 , children have likely had some experience in this type of articulatory compensation.

The current data suggest that compensatory articulation may be present relatively early in development and therefore suggest a rather minimal role of language experience necessary for developing compensatory articulation abilities. However, by age 4, children have already had a good deal of language experience. Further studies might assess these abilities in even younger children. A recent study by Smith and McLean-Muse (1987b) has even suggested the possibility that young children demonstrate greater interarticulatory reciprocity, hence greater compensatory articulation, than do adults. It should be noted, however, that the present experiment addresses only achievement of an hypothesized acoustic target, not the articulatory gestures involved in reaching that target. Results of articulatory studies may thus reveal incomplete or selective compensation in adults and children (or different patterns of compensation in the two groups), while acoustic or perceptual goals are still attained by both groups of speakers. 


\section{ACKNOWLEDGMENTS}

The authors would like to thank Clarissa Kripke for assistance in data collection, and Dr. Philip Lieberman and Dr. Paula Tallal for their help. We are also grateful to several anonymous reviewers for their helpful comments. This research was supported, in part, by Grant \#NS 15123 to Brown University and by Grant NS 22343-01 to the San Diego Center for Neurodevelopmental Studies.

${ }^{1}$ A completely random order was initially attempted with two subjects. However, this procedure proved overly taxing for the 4-year-old subjects. For this reason, these data were discarded, and the new procedure was adopted.

${ }^{2}$ In contrast to the Oller and MacNeilage (1983) study that utilized spectrographic analyses, we chose to compute LPC spectra to improve resolution, especially when dealing with high fundamental frequencies associated with children's speech.

${ }^{3}$ Incomplete compensation does not preclude production of an identifiable version of the target speech sound. Rather, the suggestion is that enough compensatory adjustment has occurred to achieve the target production, but that, if judged subjectively, the token may be considered less than perfect (cf. Flege et al., 1988).

Abbs, J. (1986). "Invariance and variability in speech production: a distinction between linguistic intent and its neuromotor implementation," in Invariance and Variability in Speech Processes, edited by J. Perkell and D. Klatt (Erlbaum, Hillsdale, NJ).

Borden, G. (1979). "An interpretation of feedback in established and developing speech," in Speech and Language: Advances in Basic Research, Vol. 3, edited by N. Lass (Academic, New York), pp. 223-242.

Eguchi, S., and Hirsh, I. (1969). "Development of speech sounds in children," Acta Oto-Laryngol. Suppl. 257.

Flanagan, J. (1955). "A difference limen for vowel formant frequency," J. Acoust. Soc. Am. 27, 613-617.

Flege, J., Fletcher, S., and Homiedan, A. (1988). "Compensating for a bite block in /s/ and / $/$ production: Palatographic, acoustic, and perceptual data," J. Acoust. Soc. Am. 83, 212-228.

Folkins, J., and Linville, R. (1983). "The effects of varying lower-lip displacement on upper-lip movements: implications for the coordination of speech movements," J. Speech Hear. Res. 26, 209-217.

Folkins, J., and Zimmermann, G. (1982). "Lip and jaw interaction during speech: Responses to perturbation of lower-lip movement prior to bilabial closure," J. Acoust. Soc. Am. 71, 1225-1233.

Fowler, C., and Turvey, M. (1980). "Immediate compensation in biteblock speech," Phonetica 37, 306-326.

Gay, T., Lindblom, B., and Lubker, J. (1981). "Production of bite block vowels: Acoustic equivalence by selective compensation," J. Acoust. Soc. Am. 69, 802-810.

Gibson, A., and McPhearson, L. (1980). "Production of bite-block vowels by children," PERLUS Report II, 26-43.

Gracco, V., and Abbs, J. (1985). "Dynamic control of the perioral system during speech: kinematic analyses of autogenic and nonautogenic sensorimotor processes," J. Neurophysiol. 54, 418-432.

Hebb, D. (1949). The Organization of Behavior: A Neurophysiological Theory (Wiley, New York).

Hughes, O., and Abbs, J. (1976). "Labial-mandibular coordination in the production of speech: implications for the operation of motor equivalence," Phonetica 33, 199-221.

Kelso, J., and Tuller, B. (1983a). "Compensatory articulation under conditions of reduced afferent information: a dynamic formulation," J. Speech Hear. Res. 26, 217-223.

Kelso, J., and Tuller, B. (1983b). "Converging evidence in support of common dynamical principles for speech and movement coordination," Haskins Lab. Stat. Rep. Speech Res. SR-74/75.

Kewley-Port, D., and Preston, M. (1974). "Early apical stop production: a voice onset time analysis," J. Phon. 2, 195-210.

Konefal, J., Fokes, J., and Bond, Z. (1982). "Children's use of syntactic vowel duration," J. Phon. 10, 361-366.

Kubaska, C., and Keating, P. (1981). "Word duration in early child speech," J. Speech Hear. Res. 24, 615-621.

Lindblom, B., Lubker, J., and Gay, T. (1979). "Formant frequencies of some fixed-mandible vowels and a model of speech motor programming by predictive simulation," J. Phon. 7, 147-161.

Lindbloom, B., and Sundberg, J. (1970). "Acoustical consequences of lip, tongue, jaw, and larynx movement," J. Acoust. Soc. Am. 50, 1166-1171.

Lubker, J. (1979). "The reorganization times of bite-block vowels," Phonetica 36, 273-293.

Macken, M., and Barton, D. (1980). "The acquisition of the voicing contrast in English: a study of voice onset time in word-initial stop consonants," J. Child Lang. 7, 41-74.

MacNeilage, P. (1970). "Motor control of serial ordering of speech," Psychol. Rev. 77, 182-196.

Netsell, R. (1985). "Construction and use of a bite-block for the evaluation and treatment of speech disorders," J. Speech Hear. Disord. 50, 103-106.

Oller, D. K., and MacNeilage, P. (1983). "Development of speech production: perspectives from natural and perturbed speech," in The Production of Speech, edited by P. MacNeilage (Springer, New York).

Perkell, J. (1980). "Phonetic features and the physiology of speech production," in Language Production, edited by B. Butterworth (Academic, London).

Peterson, G., and Barney, H. (1952). "Control methods used in a study of the vowels," J. Acoust. Soc. Am. 24, 175-184.

Pickett, J. (1980). The Sounds of Speech Communication (Pro-Ed, Austin, TX).

Sereno, J., Baum, S., Marean, G., and Lieberman, P. (1987). “Acoustic analyses and perceptual data on anticipatory labial coarticulation in adults and children," J. Acoust. Soc. Am. 81, 512-519.

Sharkey, S., and Folkins, J. (1985). "Variability of lip and jaw movements in children and adults: implications for the development of speech motor control," J. Speech Hear. Res. 28, 3-15.

Smith, B. (1977). "Temporal aspects of English speech production: a developmental perspective," J. Phon. 6, 37-69.

Smith, B., and McLean-Muse, A. (1987a). "Effects of rate and bite block manipulations on kinematic characteristics of children's speech," J. Acoust. Soc. Am. 81, 747-754.

Smith, B., and McLean-Muse, A. (1987b). "An investigation of motor equivalence in the speech of children and adults," J. Acoust. Soc. Am. 82, 837-842.

Watkin, K., and Fromm, D. (1984). "Labial coordination in children: Preliminary considerations," J. Acoust. Soc. Am. 75, 629-632. 